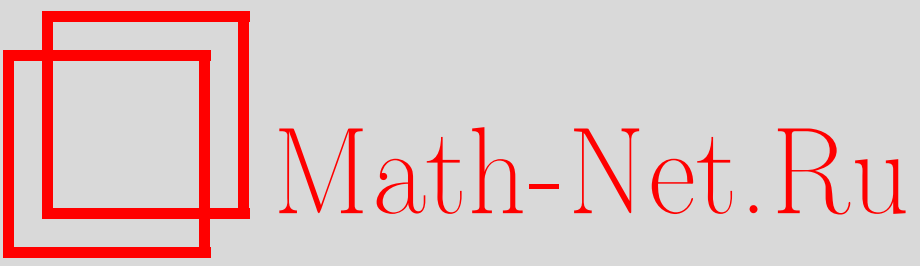

Э. А. Аринштейн, Теория возмущений для матриц плотности и термодинамического потенциала квантовой системы, ТМФ, 2002, том 130, номер 1, 54-63

DOI: https://doi.org/10.4213/tmf290

Использование Общероссийского математического портала Math-Net.Ru подразумевает, что вы прочитали и согласны с пользовательским соглашением

http://www . mathnet.ru/rus/agreement

Параметры загрузки:

IP : 3.89.197.203

26 апреля 2023 г., 14:30:34 
ТЕОРЕТИЧЕСКАЯ

И МАТЕМАТИЧЕСКАЯ

ФИЗИКА

Том 130, № 1

январь, 2002

(C) 2002 г.

Э. А. Аринштейн*

\section{ТЕОРИЯ ВОЗМУЩЕНИЙ ДЛЯ МАТРИЦ ПЛОТНОСТИ И ТЕРМОДИНАМИЧЕСКОГО ПОТЕНЦИАЛА КВАНТОВОЙ СИСТЕМЫ}

Разложение термодинамического потенциала квантовой системы, справедливое при высоких температурах, получено методом производящего функционала для матричных элементов операторов вторичного квантования. Этот метод позволяет выделить неприводимые части матриц, в том числе частичных матриц плотности. Получено уравнение для полной унарной матрицы плотности, эквивалентное вариационному принципу для термодинамического потенциала. Таким образом, термодинамические функции и матрица плотности определяются в рамках единой вариационной задачи.

В последнее время возрос интерес к исследованию макроскопических квантовых эффектов при высоких температурах. Метод квантовых групповых разложений для исследования термодинамических свойств квантовых систем был предложен Блохом и Доминисисом [1]-[3] по аналогии с методом групповых разложений в классической статистической физике. Частичное суммирование этих разложений, проведенное Ли и Янгом [4], позволило рассмотреть систему частиц с сильным отталкиванием на малых расстояниях (газ твердых сфер) с точностью до членов второго порядка. Этим же методом можно найти первые члены разложения частичных матрищ плотности [5]. Систематический метод построения подобных разложений с любой степенью точности и приближенных интегральных уравнений для частичных матриц плотности, аналогичных интегральным уравнениям для классических частичных функций распределения, основан на методе производящего функционала.

\section{1. МЕТОД ПРОИЗВОДЯШЕГО ФУНКЦИОНАЛА}

Операторы рождения и поглощения частиц в одночастичных состояниях, характеризуемых набором дискретных или непрерывных квантовых чисел $i$, обозначим, как обычно, $a^{+}(i)$ и $a(i)$. Эти операторы подчиняются стандартным перестановочным соотношениям - коммутационным или антикоммутационным.

Введем производяший функционал для матричных элементов произвольного оператора в представлении вторичного квантования. Пусть $\xi^{*}(i)$ и $\lambda(i)$ - основные функции,

\footnotetext{
* Тюменский государственный университет, Тюмень, Россия. E-mail: earin@tsu.tmn.ru
} 
зависящие от того же набора квантовых чисел, что и операторы рождения и поглощения. В случае статистики Ферми эти функции являются образующими грассмановой алгебры [6], антикоммутируюшими между собой и с операторами рождения и поглощения ферми-частиц. Скалярное произведение в гильбертовом пространстве одночастичных состояний обозначим, как обычно, $\left(\xi^{*} \lambda\right)$. Остальные свойства пространства основных функций также определяются стандартным образом. Возможно рассмотрение системы, состоящей из различных частиш, в том числе из частищ различного типа.

Пусть $A$ - оператор некоторой физической величины в представлении вторичного квантования. Функционал

$$
A\left(\xi^{*}, \lambda\right)=\left\langle 0\left|e^{\left(\xi^{*} a\right)} A e^{\left(a^{+} \lambda\right)}\right| 0\right\rangle
$$

является производяшим для матричных элементов оператора $A$. Обычно оператор может быть представлен в нормальной форме:

$$
A=\sum_{m, n}\left(\prod_{\{m\}} a^{+}(i) \tilde{A}(\{m\},\{n\}) \prod_{\{n\}} a(j)\right) .
$$

Величины $\tilde{A}(\{m\},\{n\})$ (функции от наборов квантовых чисел) являются коэффициентами нормального разложения. Иногда из этих коэффициентов выделяется комбинаторный множитель $(m ! n !)^{-1 / 2}$. С учетом соотношений

$$
e^{\left(\xi^{*} a\right)} a^{+}(i) e^{-\left(\xi^{*} a\right)}=a^{+}(i)+\xi^{*}(i), \quad e^{-\left(a^{+} \lambda\right)} a(i) e^{\left(a^{+} \lambda\right)}=a(i)+\lambda(i),
$$

которые легко установить разложением по функциям $\xi^{*}$ и $\lambda$, получим

$$
A\left(\xi^{*}, \lambda\right)=e^{\left(\xi^{*} \lambda\right)} \sum_{m, n}\left(\prod_{\{m\}} \xi^{*}(i) \tilde{A}(\{m\},\{n\}) \prod_{\{n\}} \lambda(j)\right) .
$$

Таким образом, функционал

$$
\tilde{A}\left(\xi^{*}, \lambda\right)=e^{-\left(\xi^{*} \lambda\right)} A\left(\xi^{*}, \lambda\right)
$$

является производящим для коэффициентов нормального разложения.

Вводя функциональную свертку

$$
A_{1}(\xi) \otimes A_{2}\left(\xi^{*}\right)=\left.A_{1}\left(\frac{\delta}{\delta \xi^{*}}\right) A_{2}\left(\xi^{*}\right)\right|_{\xi^{*}=0}=\left.A_{1}(\xi) A_{2}\left(\overleftarrow{\frac{\delta}{\delta \xi}}\right)\right|_{\xi=0}
$$

представим оператор $A$ в виде

$$
A=e^{\left(a^{+} \xi\right)} \otimes \tilde{A}\left(\xi^{*}, \lambda\right) \otimes e^{\left(\lambda^{*} a\right)},
$$

а след оператора в виде

$$
\operatorname{Sp} A=A\left(\xi^{*}, \xi\right) \otimes,
$$


причем в случае статистики Ферми немая функция $\xi^{*}$ при свертке переносится циклически направо, а функция $\xi$ - налево. Заметим, что немая функция всегда сворачивается со своей сопряженной.

Используя представление (6), можно легко установить соответствие

$$
A=A_{1} A_{2} \Longleftrightarrow A\left(\xi^{*}, \lambda\right)=A_{1}\left(\xi^{*}, \gamma\right) \otimes A_{2}\left(\gamma^{*}, \lambda\right) .
$$

Представление (1) производящего функционала эквивалентно представлению, введенному Березиным [6], однако для некоторых приложений оно может оказаться удобнее. Видно также, что оно связано с разложением по когерентным состояниям.

Производящий функционал для неприводимых частей матричных элементов оператора $A$ определяется соотношением

$$
A\left(\xi^{*}, \lambda\right)=e^{B\left(\xi^{*}, \lambda\right)}
$$

Если представить функционалы, входяшие в выражения (7) и (8), через их неприводимые части, то свертка разобъется на произведение независимых (несвязанных) частей. Обычный подсчет множителей каждого типа приводит к теореме о связанных частях для следа оператора

$$
\operatorname{Sp} A=e^{B\left(\xi^{*}, \xi\right)} \otimes=\exp \left(e^{B\left(\xi^{*}, \xi\right)} \otimes\right)_{\mathrm{c}}
$$

и для свертки двух функционалов

$$
e^{B_{1}\left(\xi^{*}, \gamma\right)} \otimes e^{B_{2}\left(\gamma^{*}, \lambda\right)}=\exp \left(e^{B_{1}\left(\xi^{*}, \gamma\right)} \otimes e^{B_{2}\left(\gamma^{*}, \lambda\right)}\right)_{\mathrm{c}} .
$$

В случае смешанного состояния, характеризуемого статистическим оператором $\rho$, среднее физической величины определяется как $\langle A\rangle=\operatorname{Sp} \rho A$. Среднее значение физической величины, в представлении вторичного квантования имеющей вид

$$
\prod_{\{m\}} a^{+}(i) \tilde{A}(\{m\},\{n\}) \prod_{\{n\}} a(j)
$$

принимает значение

$$
\begin{aligned}
\sum_{m, n} & \operatorname{Sp} \rho \prod_{\{m\}} a^{+}(i) \tilde{A}(\{m\},\{n\}) \prod_{\{n\}} a(j)= \\
\quad= & \sum_{\{m\},\{n\}} R_{n, m}(\{n\},\{m\}) \tilde{A}_{m, n}(\{m\},\{n\}),
\end{aligned}
$$

где

$$
R_{n, m}(\{n\},\{m\})=\operatorname{Sp} \rho \prod_{\{m\}} a^{+}(i) \prod_{\{n\}} a(j)=\operatorname{Sp} \prod_{\{n\}} a(j) \rho \prod_{\{m\}} a^{+}(i)
$$

- матрища плотности порядка $n, m$. В обшем случае

$$
\begin{aligned}
\langle A\rangle & =\operatorname{Sp} \rho A=\operatorname{Sp} \rho e^{\left(a^{+} \xi\right)} \otimes \tilde{A}\left(\xi^{*}, \lambda\right) \otimes e^{\left(\lambda^{*} a\right)}= \\
& =\left(\operatorname{Sp} e^{\left(\lambda^{*} a\right)} \rho e^{\left(a^{+} \xi\right)}\right) \otimes \tilde{A}\left(\xi^{*}, \lambda\right) \otimes=R\left(\lambda^{*}, \xi\right) \otimes \tilde{A}\left(\xi^{*}, \lambda\right) \otimes .
\end{aligned}
$$


Таким образом, функционал

$$
R\left(\xi^{*}, \lambda\right)=\operatorname{Sp} e^{\left(\xi^{*} a\right)} \rho e^{\left(a^{+} \lambda\right)}=\sum_{\{m\},\{n\}} \frac{1}{m ! n !} \prod_{\{m\}} \xi^{*}(i) \prod_{\{n\}} \lambda(j) R_{m, n}(\{m\},\{n\})
$$

является производящим для частичных матриц плотности. Вводя представление

$$
R\left(\xi^{*}, \lambda\right)=e^{\left(\xi^{*} \rho_{11} \lambda\right)+G\left(\xi^{*}, \lambda\right)}
$$

где $\rho_{11}$ - унарная матрица плотности, определим производящий функционал $G\left(\xi^{*}, \lambda\right)$ корреляционных матриц, являющихся неприводимыми частями многочастичных матриц плотности.

Из определения матриц плотности (13) следуют соотношение

$$
\left\langle\rho_{m+k, n+l}\right\rangle_{\{k\},\{l\}}=\rho_{m, n}
$$

и их нормировка $\left\langle\rho_{m, n}\right\rangle_{\{m\},\{n\}}=1$.

\section{2. МАТРИЦЫ ПЛОТНОСТИ ИДЕАЛЬНЫХ ГАЗОВ}

Статистический оператор равновесного идеального газа имеет вид

$$
\rho_{0}=e^{\beta\left(\Omega_{0}-\left(a^{+} T a\right)\right)}, \quad T=H_{0}-\mu .
$$

Производящий функционал матриц плотности в этом случае можно найти исходя из перестановочных соотношений между операторами $\left(a^{+} T a\right)$ и $\left(\xi^{*} a\right)$.

Разложением по степеням $\beta$ легко установить соотношение

$$
e^{\beta\left(a^{+} T a\right)}\left(\xi^{*} a\right) e^{-\beta\left(a^{+} T a\right)}=\left(\xi^{*} e^{-\beta T} a\right) .
$$

Тогда

$$
\begin{aligned}
R_{0}\left(\xi^{*}, \lambda\right) & =\operatorname{Sp} e^{\left(\xi^{*} a\right)} \rho_{0} e^{\left(a^{+} \lambda\right)}=\operatorname{Sp} \rho_{0} e^{\beta\left(a^{+} T a\right)} e^{\left(\xi^{*} a\right)} e^{-\beta\left(a^{+} T a\right)} e^{\left(a^{+} \lambda\right)}= \\
& =\operatorname{Sp} \rho_{0} \exp \left(\xi^{*} e^{-\beta T} a\right) e^{\left(a^{+} \lambda\right)}= \\
& =\exp \left(\xi^{*} e^{-\beta T} \lambda\right) \operatorname{Sp} \rho_{0} e^{\left(a^{+} \lambda\right)} \exp \left(\xi^{*} e^{-\beta T} a\right) .
\end{aligned}
$$

В случае статистики Бозе, пользуясь правилом циклической перестановки операторов под знаком следа, отсюда сразу же получим уравнение

$$
R_{0}\left(\xi^{*}, \lambda\right)=\exp \left(\xi^{*} e^{-\beta T} \lambda\right) R_{0}\left(\xi^{*} e^{-\beta T}, \lambda\right),
$$

или после замены $\xi^{*} e^{-\beta T}$ на новую произвольную функцию $\xi^{*}$ уравнение

$$
R_{0}\left(\xi^{*} e^{\beta T}, \lambda\right)=e^{\left(\xi^{*} \lambda\right)} R_{0}\left(\xi^{*}, \lambda\right)
$$


В случае статистики Ферми правило циклической перестановки относится только к операторам $a$ и $a^{+}$, тогда как множители $\xi^{*}$ и $\lambda$ необходимо переставлять с учетом их антикоммутативности. Перестановка этих множителей приводит к уравнению

$$
R_{0}\left(\xi^{*}, \lambda\right)=\exp \left(\xi^{*} e^{-\beta T} \lambda\right) R_{0}\left(\xi^{*} e^{-\beta T},-\lambda\right)
$$

или

$$
R_{0}\left(\xi^{*} e^{\beta T} \lambda\right)=e^{\left(\xi^{*} \lambda\right)} R_{0}\left(\xi^{*},-\lambda\right)
$$

Переходя к неприводимым частям функционала $R_{0}$ и объединяя оба случая, получим уравнение

$$
\left(\xi^{*}\left(e^{\beta T} \pm 1\right) \rho_{1}^{0} \lambda\right)+G_{0}\left(\xi^{*} e^{\beta T}, \lambda\right)=\left(\xi^{*} \lambda\right)+G_{0}\left(\xi^{*}, \pm \lambda\right),
$$

откуда сравнением членов одинакового порядка по $\xi^{*}$ и $\lambda$ получим для корреляционных матриц идеального газа $G_{0}\left(\xi^{*}, \lambda\right)=0$ и, произведя замену $\xi^{*}\left(e^{\beta T} \pm 1\right) \rightarrow \xi^{*}$, достаточно очевидный результат:

$$
\left(\xi^{*} \rho_{1}^{0} \lambda\right)=\left(\xi^{*} \frac{1}{e^{\beta T} \pm 1} \lambda\right)
$$

Окончательно имеем

$$
R_{0}\left(\xi^{*}, \lambda\right)=\exp \left(\xi^{*} \frac{1}{e^{\beta T} \pm 1} \lambda\right)
$$

Из (24) можно получить все многочастичные матрицы плотности идеальных газов в виде (анти)симметризованных произведений унарных матриц. При этом автоматически учитывается корреляция, определяемая квантовой статистикой.

\section{3. УЛУЧШЕННАЯ ТЕРМОДИНАМИЧЕСКАЯ ТЕОРИЯ ВОЗМУЩЕНИЙ}

Статистическая сумма системы взаимодействующих частиц имеет вид

$$
Z=\operatorname{Sp} e^{-\beta(H-\mu N)}=Z_{0} \operatorname{Sp} \rho_{0} S
$$

где $S=e^{\beta H_{0}} e^{-\beta H}-$ оператор температурного рассеяния Мацубары [7]. Оператор Мацубары определен для гамильтонианов, имеющих ограниченный снизу спектр, в том числе и для взаимодействия, включающего сильное отталкивание на малых расстояниях (твердую сердшевину).

В случае идеального газа $S=1$, соответствующий производящий функционал равен $S_{0}\left(\xi^{*}, \lambda\right)=e^{\left(\xi^{*} \lambda\right)}$ и, следовательно, $\widetilde{S}_{0}\left(\xi^{*}, \lambda\right)=1$.

Введем неприводимые части функционала $S$ :

$$
S\left(\xi^{*}, \lambda\right)=e^{\left(\xi^{*} \lambda\right)} \widetilde{S}\left(\xi^{*}, \lambda\right)=e^{\left(\xi^{*} \lambda\right)+u\left(\xi^{*}, \lambda\right)} .
$$

В случае гамильтониана, сохраняющего число частиц,

$$
u\left(\xi^{*}, \lambda\right)=u_{2}\left(\xi^{*}, \lambda\right)+u_{3}\left(\xi^{*}, \lambda\right)+\cdots+u_{k}\left(\xi^{*}, \lambda\right)+\cdots
$$


т.е. функционал распадается на двух-, трех-, $k$-частичные неприводимые, диагональные по числу частиц части, имеюшие соответствуюший одинаковый порядок по функциям $\xi^{*}$ и $\lambda$. Двухчастичная неприводимая часть совпадает с бинарным ядром, полученным Янгом и Ли суммированием бесконечной подпоследовательности членов ряда теории возмушений, и находится в явном виде из решения задачи двух тел:

$$
u_{2}\left(\xi^{*}, \lambda\right)=\frac{1}{2}\left(\xi^{*}(1) \xi^{*}(2)\left(e^{\beta H_{0}^{(2)}} e^{-\beta H^{(2)}}-1\right) \lambda\left(1^{\prime}\right) \lambda\left(2^{\prime}\right)\right) .
$$

Для определения старших неприводимых частей требуется решение соответствующих $k$-частичных задач либо разложение по бинарным ядрам последовательно для $k=3$, $4, \ldots$. В обшем случае для гамильтониана, не сохраняюшего число частищ, например фононов, функционал $u$ будет содержать недиагональные по числу частиц члены вида $u_{i, k}$.

Более детальное рассмотрение неприводимых частей оператора Мацубары приведено в приложении.

Используя представление $(28)$ и соотношение $e^{\left(\xi^{*}\left(\delta / \delta \lambda^{*}\right)\right)} A\left(\lambda^{*}\right)=A\left(\xi^{*}+\lambda^{*}\right)$, получим

$$
\begin{aligned}
Z & =Z_{0} R_{0}\left(\xi^{*}, \lambda\right) \otimes \widetilde{S}\left(\lambda^{*}, \xi\right)=Z_{0} e^{\left(\xi^{*} \rho_{1}^{0} \lambda\right)} \otimes e^{u\left(\lambda^{*}, \xi\right)}= \\
& =Z_{0} \exp \left(e^{u\left(\xi^{*} \rho_{1}^{0}, \xi\right)} \otimes\right)_{\mathrm{c}}=Z_{0} \exp \left(\exp \left(u\left(\xi^{*} e^{-\beta(E-\mu)}\left(1 \pm \rho_{1}^{0}\right), \xi\right)\right) \otimes\right)_{\mathrm{c}}
\end{aligned}
$$

откуда

$$
\Omega=\Omega_{0}-\frac{1}{\beta}\left(e^{u\left(\xi^{*} \rho_{1}^{0}, \xi\right)} \otimes\right)_{\mathrm{c}}=\Omega_{0}-\frac{1}{\beta}\left(\exp \left(u\left(\xi^{*} e^{-\beta(E-\mu)}\left(1 \pm \rho_{1}^{0}\right), \xi\right)\right) \otimes\right)_{\mathrm{c}} .
$$

Заметим, что функционал $\tilde{u}\left(\xi^{*}, \lambda\right)=u\left(\xi^{*} e^{-\beta(E-\mu)}, \lambda\right)$ определяет неприводимые части эрмитова оператора $e^{-\beta(H-\mu)}$, в отличие от функционала $u\left(\xi^{*}, \lambda\right)$, определяющего неприводимые части неэрмитова оператора Мацубары. Разложение (32) является квантовым обобшением классического разложения по степеням плотности. Ли и Янг получили первые два члена этого разложения суммированием бесконечной подпоследовательности разложения по бинарным ядрам.

Аналогичным образом получим разложение частичных матриц плотности, точнее производящего функционала этих матриц:

$$
\begin{aligned}
R\left(\xi^{*}, \lambda\right) & =\operatorname{Sp} e^{\left(\xi^{*} a\right)} \rho e^{\left(a^{+} \lambda\right)}=e^{\beta\left(\Omega-\Omega_{0}\right)} \operatorname{Sp} e^{\left(\xi^{*} a\right)} \rho_{0} S e^{\left(a^{+} \lambda\right)}= \\
& =e^{\beta\left(\Omega-\Omega_{0}\right)} \operatorname{Sp} e^{\left(\xi^{*} a\right)} \rho_{0} e^{\left(a^{+} \gamma\right)} \otimes e^{u\left(\gamma^{*}, \nu\right)} \otimes e^{\left(\nu^{*} a\right)} e^{\left(a^{+} \lambda\right)}= \\
& =e^{\beta\left(\Omega-\Omega_{0}\right)} \operatorname{Sp} e^{\left(\left(\xi^{*}+\nu^{*}\right) a\right)} \rho_{0} e^{\left(a^{+}(\gamma+\lambda)\right)} \otimes e^{u\left(\gamma^{*}, \nu+\lambda\right)} .
\end{aligned}
$$

Это приводит к разложению

$$
R\left(\xi^{*}, \lambda\right)=e^{\beta\left(\Omega-\Omega_{0}\right)+\left(\left(\xi^{*}+\nu^{*}\right) \rho_{1}^{0}(\gamma+\lambda)\right)} \otimes e^{u\left(\gamma^{*}, \nu+\lambda\right)} .
$$


Необходимо учесть, что в скалярном произведении $\left(\nu^{*} \rho_{1}^{0} \lambda\right)$ функция $\nu^{*}$ циклически перенесена направо при свертке, тогда как функция $\lambda$ остается слева. Следовательно, при явном выполнении циклического переноса справа окажется множитель $e^{\left(\lambda \rho_{1}^{0} \nu^{*}\right)}=$ $e^{ \pm\left(\nu^{*} \rho_{1}^{0} \lambda\right)}$. В итоге получим

$$
\begin{aligned}
R\left(\xi^{*}, \lambda\right)= & e^{\beta\left(\Omega-\Omega_{0}\right)+\left(\xi^{*} \rho_{1}^{0} \lambda\right)} e^{u\left(\left(\xi^{*}+\nu^{*}\right) \rho_{1}^{0}, \nu+\left(1 \pm \rho_{1}^{0}\right) \lambda\right)} \otimes= \\
= & \exp \left(\left(\xi^{*} \rho_{1}^{0} \lambda\right)+\left(e^{u\left(\left(\xi^{*}+\nu^{*}\right) \rho_{1}^{0}, \nu+\left(1 \pm \rho_{1}^{0}\right) \lambda\right)} \otimes\right)_{\mathrm{c}}-\left(e^{u\left(\nu^{*} \rho_{1}^{0}, \nu\right)} \otimes\right)_{\mathrm{c}}\right)= \\
= & \exp \left(\left(\xi^{*} \rho_{1}^{0} \lambda\right)+\left(\exp \left(u\left(\left(\xi^{*}+\nu^{*}\right) e^{-\beta(E-\mu)}\left(1 \pm \rho_{1}^{0}\right), \nu+\left(1 \pm \rho_{1}^{0}\right) \lambda\right)\right) \otimes\right)_{\mathrm{c}}-\right. \\
& \left.-\left(e^{u\left(\nu^{*} \rho_{1}^{0}, \nu\right)} \otimes\right)_{\mathrm{c}}\right)
\end{aligned}
$$

где знаки определяются, как обычно, статистикой. Это разложение всех матриц плотности является квантовым обобщением разложения частичных функций распределения по плотности и переходит в него в классическом пределе. Первые члены разложения (33) для унарной, бинарной и тройной матриц плотности были получены Исихарой [5] суммированием квантовых групповых разложений.

\section{4. ЧАСТИЧНОЕ СУММИРОВАНИЕ РЯДОВ ТЕОРИИ ВОЗМУЩЕНИЙ}

Каждый член разложений (32) и (35) можно представить диаграммой, сопоставляя неприводимой части $u_{i, k}$ вершину с $(i+k)$ линиями $\left(i\right.$ выходящих функций $\xi^{*}$ и $k$ входящих функций $\lambda$ ), а функции $\rho_{1}^{0}$ внутренние линии, объединяющие по одной выходяшей и одной входящей линии и тем самым связывающие вершины. Диаграммы разложения (35) содержат свободные (или внешние) линии - выходящие и входяшие. При использовании вершин $u\left(\xi^{*}, \lambda\right)$ выходящие линии содержат множитель $\rho_{1}^{0}$, а входящие множитель $\left(1 \pm \rho_{1}^{0}\right)$. При использовании вершин $\tilde{u}\left(\xi^{*}, \lambda\right)$ и выходящие, и входяшие свободные линии содержат одинаковый множитель $\left(1 \pm \rho_{1}^{0}\right)$.

Введем величину $M_{1}\left(1,1^{\prime}\right)$ - сумму 1 -неприводимых диаграмм, имеюших одну входящую и одну выходяшую свободные линии при условии, что диаграмма не может быть разделена на две несвязанные части удалением одной внутренней линии. Очевидно, что для пространственно однородных систем 1-неприводимая диаграмма, так же как и матрица $\rho_{1}$, в импульсном представлении диагональна. Функция $M_{1}$ связана с полной одночастичной матрицей плотности. Выделяя в разложении (35) члены первого порядка по функциям $\xi^{*}$ и $\lambda$, получим

$$
\begin{aligned}
\left(\xi^{*} \rho_{1} \lambda\right)= & \left(\xi^{*} \rho_{1}^{0} \lambda\right)+\left(\xi^{*} \rho_{1}^{0} M_{1}\left(1 \pm \rho_{1}^{0}\right) \lambda\right) \pm \\
& \pm\left(\xi^{*} \rho_{1}^{0} M_{1} \nu\right) \otimes\left(\nu^{*} \rho_{1}^{0} M_{1}\left(1 \pm \rho_{1}^{0}\right) \lambda\right)+\cdots= \\
= & \left(\xi^{*} \rho_{1}^{0} \lambda\right)+\left(\xi^{*} \frac{\rho_{1}^{0} M_{1}}{1 \pm \rho_{1}^{0} M_{1}}\left(1 \pm \rho_{1}^{0}\right) \lambda\right)=\left(\xi^{*} \frac{1+M_{1}}{e^{\beta(E-\mu)} \pm\left(1+M_{1}\right)} \lambda\right)
\end{aligned}
$$

Общий член ряда имеет вид $( \pm 1)^{n-1}\left(\xi^{*}\left(\rho_{1}^{0} M_{1}\right)^{n}\left(1 \pm \rho_{1}^{0}\right) \lambda\right)$. Комбинаторный множитель равен $n$ ! и сокрашается с таким же знаменателем, возникаюшим при разложении 
экспоненты; знаковый множитель $( \pm 1)^{n-1}$ возникает при переносе справа и перестановке каждой из $n-1$ промежуточных сворачиваемых функций $\nu_{k}^{*}$ с несколькими парами, входяшими в скалярные произведения $\left(\nu_{j}^{*} \rho_{1}^{0} M_{1} \nu_{j-1}\right)$, что не дает изменения знака, и функцией $\nu_{k-1}^{*}$, входящей в то же скалярное произведение, что и функция $\nu_{k}^{*}$.

Из тождества $e^{u\left(\nu^{*} \rho_{1}^{0}, \nu\right)} \otimes_{\nu}=e^{u\left(\left(\xi^{*}+\nu^{*}\right) \rho_{1}^{0},(\nu+\xi)\right)} \otimes_{\nu, \xi}$ следует, что замыкание свободных линий в диаграммном разложении матрищ плотности (с исключением лишних множителей) дает в сумме диаграммное разложение термодинамического потенциала. Таким образом, диаграммы, входящие в разложение матрищ плотности, в частности 1-неприводимые диаграммы, являются элементами, из которых строятся диаграммы, дающие разложение термодинамического потенциала. Это позволяет выделить в разложении (32) диаграммы двух основных типов.

Выделим, во-первых, вклад 0-неприводимых (скелетных) диаграмм $M_{0}\left(\rho_{1}^{0}\right)$, не содержаших во внутренних линиях 1-неприводимых частей, и, во-вторых, вклад циклических диаграмм, образованных двумя или более 1-неприводимыми диаграммами:

$$
\sum_{n \geqslant 2} \frac{( \pm 1)^{n-1}}{n}\left(\xi^{*}\left(\rho_{1}^{0} M_{1}\right)^{n} \xi\right) \otimes=\mp\left(\xi^{*} \ln \left(1 \mp \rho_{1}^{0} M_{1}\right) \xi\right) \otimes-\left(\xi^{*} \rho_{1}^{0} M_{1} \xi\right) \otimes .
$$

Для учета вклада остальных диаграмм следует заменить в диаграммах этих двух типов внутренние линии $\rho_{1}^{0}$ на линии

$$
l=\sum_{n \geqslant 0}\left( \pm \rho_{1}^{0} M_{1}\right)^{n} \rho_{1}^{0}=\frac{\rho_{1}^{0}}{1 \mp \rho_{1}^{0} M_{1}}=\frac{\rho_{1}}{1+M_{1}}=e^{-\beta(E-\mu)}\left(1 \pm \rho_{1}\right) .
$$

Таким образом, разложение термодинамического потенциала системы с учетом явного выражения для термодинамического потенциала идеального газа, содержащее только скелетные диаграммы с внутренними линиями (38), примет вид

$$
\begin{aligned}
\beta \Omega= & \pm\left(\xi^{*} \ln \left(1 \mp e^{-\beta(E-\mu)}\right) \xi\right) \otimes \pm \\
& \pm\left(\xi^{*} \ln \left(1 \mp \rho_{1}^{0} M_{1}(l)\right) \xi\right) \otimes+\left(\xi^{*} l M_{1}(l) \xi\right) \otimes-M_{0}(l) .
\end{aligned}
$$

\section{5. ВАРИАЦИОННЫЙ ПРИНЦИП ДЛЯ ТЕРМОДИНАМИЧЕСКОГО ПОТЕНЦИАЛА}

Суммирование 1-неприводимых вкладов во внутренних линиях диаграмм разложения (36) (перенормировка внутренних линий) преврашает это разложение в уравнение для полной унарной матрицы плотности, так как перенормированные линии зависят от нее. Записывая это уравнение в виде

$$
\ln \rho_{1}-\ln \left(1 \pm \rho_{1}\right)+\beta(E-\mu)-\ln \left(1+M_{1}(l)\right)=0
$$

и скалярно умножая на $\delta \rho_{1}$, найдем, что оно эквивалентно вариационному принципу для функционала

$$
\begin{aligned}
\beta \Omega= & \left(\xi^{*}\left\{\rho_{1} \ln \rho_{1} \mp\left(1 \pm \rho_{1}\right) \ln \left(1 \pm \rho_{1}\right)+\rho_{1} \beta(E-\mu)\right\} \xi\right) \otimes- \\
& -\left(\xi^{*}\left\{\rho_{1} \ln \left(1+M_{1}\right)-l M_{1}\right\} \xi\right) \otimes-M_{0}(l) .
\end{aligned}
$$


Нетрудно проверить, что на экстремали (40) функционал (41) совпадает с термодинамическим потенциалом (39).

Таким образом, определение термодинамического потенциала и полной унарной матрицы плотности сводится к единой вариационной задаче для функционала (39). В случае системы частиц одного сорта с гамильтонианом, сохраняющим число частиц, имеем $M_{1}=\delta M_{0} / \delta l$. В случае системы, содержащей частишы нескольких сортов, функция $M_{1}$ определяется для каждого сорта частиц аналогично.

В работе [8] функционал вариационной задачи был найден с точностью до трех членов в виде разложения по функциям $\tilde{l}=\rho_{1}$, без выделения 1-неприводимых диаграмм, что привело к увеличению числа диаграмм.

Полагая в соответствии с $(36)$ и $(38)$

$$
\rho_{1}=\left(e^{\beta(E-\mu)-\omega} \mp 1\right)^{-1}, \quad l=\left(e^{\beta(E-\mu)} \mp e^{\omega}\right)^{-1},
$$

получим

$$
\beta \Omega= \pm\left(\xi^{*} \ln \left(1 \mp e^{-\beta(E-\mu)+\omega}\right) \xi\right) \otimes+\left(\xi^{*}\left(\omega-\ln \left(1+M_{1}\right)\right) \rho_{1} \xi\right) \otimes+\left(\xi^{*} l M_{1} \xi\right) \otimes-M_{0}
$$

При этом сдвиг уровня $\omega$ определяется как функция от $E$ из вариационного принципа для термодинамического потенциала.

Очевидно, что разложение по связанным диаграммам приводит в случае пространственно однородной системы к линейной зависимости термодинамического потенциала от объема. Кроме того, видно, что в случае ферми-системы сушественной является сравнительно узкая область энергий вблизи уровня Ферми (с учетом сдвига).

ПРИЛОЖКННИЕ

\section{Теория возмущений для неприводимых частей оператора Мацубары}

Из уравнения Блоха для равновесного статистического оператора

$$
\frac{\partial \rho}{\partial \beta}=-H \rho, \quad H=T+V
$$

следует уравнение

$$
\frac{\partial S}{\partial \beta}=-e^{\beta T} V e^{-\beta T} S
$$

для оператора Мацубары. Выражая входящие сюда операторы через соответствующие производящие функционалы, получим уравнение

$$
\begin{aligned}
e^{\left(\xi^{*} \lambda\right)} \frac{\partial \widetilde{S}}{\partial \beta} & =-\widetilde{V}\left(\xi^{*} e^{\beta T} ; e^{-\beta T} \nu\right) e^{\left(\xi^{*} \nu\right)} \otimes e^{\left(\nu^{*} \lambda\right)} \widetilde{S}\left(\nu^{*}, \lambda \mid \beta\right)= \\
& =-e^{\left(\xi^{*} \lambda\right)} \widetilde{V}\left(\xi^{*} e^{\beta T} ; e^{-\beta T}(\nu+\lambda)\right) \otimes \widetilde{S}\left(\nu^{*}+\xi^{*}, \lambda \mid \beta\right)= \\
& =-e^{\left(\xi^{*} \lambda\right)} \widetilde{V}\left(\xi^{*} e^{\beta T} ; e^{-\beta T}\left(\lambda+\frac{\delta}{\delta \xi^{*}}\right)\right) \widetilde{S}\left(\xi^{*}, \lambda\right) .
\end{aligned}
$$


Наконец, используя представление функционала $\widetilde{S}$ через производящий функционал $u$ неприводимых частей и соотношение

$$
e^{-u} \frac{\delta}{\delta \xi^{*}} e^{u}=\frac{\delta}{\delta \xi^{*}}+\frac{\delta u}{\delta \xi^{*}}
$$

придем к уравнению

$$
\frac{\partial u}{\partial \beta}=-\widetilde{V}\left(\xi^{*} e^{\beta T} ; e^{-\beta T}\left(\lambda+\frac{\delta u}{\delta \xi^{*}}+\frac{\delta}{\delta \xi^{*}}\right)\right)
$$

От этого дифференциального уравнения с учетом начального условия $\left.u\right|_{\beta=0} \equiv$ $u\left(\xi^{*}, \lambda \mid 0\right)=0$ легко перейти к интегральному уравнению

$$
u\left(\xi^{*}, \lambda \mid \beta\right)=-\int_{0}^{\beta} \widetilde{V}\left(\xi^{*} e^{\tau T} ; e^{-\tau T}\left(\lambda+\frac{\delta u\left(\xi^{*}, \lambda \mid \tau\right)}{\delta \xi^{*}}+\frac{\delta}{\delta \xi^{*}}\right) \mid \tau\right) d \tau .
$$

Полагая в нулевом приближении $u=0$, придем путем последовательных итераций к высокотемпературному разложению неприводимых частей матричных элементов оператора Мацубары. В случае взаимодействия, включающего интенсивное отталкивание на малых расстояниях, в качестве исходного приближения для построения итерационной процедуры следует взять бинарное ядро Ли и Янга. Легко заметить, что в случае, когда возмущение имеет характер полинома по свободным полям, правые части уравнений (45), (46) являются полиномами той же степени по неприводимым частям. Действительно, в отсутствие других множителей справа от произведения двух типичных комбинаций, входящих в эти выражения, получим

$$
\left(\lambda+\frac{\delta u}{\delta \xi^{*}}+\frac{\delta}{\delta \xi^{*}}\right)_{i}\left(\lambda+\frac{\delta u}{\delta \xi^{*}}+\frac{\delta}{\delta \xi^{*}}\right)_{k}=\lambda_{i} \lambda_{k}+\lambda_{i} \frac{\delta u}{\delta \xi_{k}^{*}}+\lambda_{k} \frac{\delta u}{\delta \xi_{i}^{*}}+\frac{\delta u}{\delta \xi_{i}^{*}} \frac{\delta u}{\delta \xi_{k}^{*}}+\frac{\delta^{2} u}{\delta \xi_{i}^{*} \delta \xi_{k}^{*}} .
$$

Произведение большего числа таких сомножителей раскрывается аналогично.

\section{Список литературы}

[1] C. Bloch, C. De Dominicis. Nucl. Phys. 1958. V. 7. P. 495; 1959. V. 10. P. 509

[2] C. Balian, C. Bloch, C De Dominicis. Nucl. Phys. 1961. V. 25. P. 529; V. 27. P. 294.

[3] C. Bloch. Stud. Stat. Mech. 1965. V. 3. P. 1.

[4] C. D. Lee, C. N. Yang. Phys. Rev. 1959. V. 113. № 5. P. 1165; V. 116. № 1. P. 25.

[5] A. Isichara. Progr. Theor. Phys. Suppl. 1969. V. 44. P. 1; Phys. Rev. 1968. V. 172. P. 166.

[6] Ф. А. Березин. Метод вторичного квантования. М.: Наука, 1965.

[7] T. Matsubara. Progr. Theor. Phys. 1956. V. 14. № 4. P. 351.

[8] Э. А. Аринштейн, Д. М. Гитман. Изв. вузов. Физика. 1968. № 10. С. 146; 1971. № 9. C. 98. 